respectively), reported that they did not use the recommended PPE at least once during the study. Forgetfulness (26.1\% and $27.3 \%$, respectively) and time constrains $(23.1 \%$ and $21.1 \%$, respectively) were the primary causes for not using the recommended PPE. At the time of the conference, data from the second round of questionnaires (response rates of $26.1 \%$ and $41.6 \%$, respectively, corresponding to 6,816 and 3,959 participants respectively) will also be available for presentation.

Conclusion One fifth of Danish healthcare workers have been in a situation where PPE was recommended but not used. Time constrains and forgetfulness are important reasons for this. Healthcare workers not wearing the recommended PPE increases the risk of the healthcare workers becoming infected with COVID-19, and is harmful to the performance of the healthcare system.

\section{0-168 COVID-19 INFECTION AND MENTAL WELLNESS IN A CANADIAN COHORT STUDY OF HEALTHCARE WORKERS}

${ }^{1}$ Nicola Cherry, France Labrèche, Anil Adisesh, Igor Burstyn, Vijay Kumar Chattu, Quentin Durand-Moreau, Jean-Michel Galarneau, Trish Mhonde, Shannon Ruzycki, Tansi Summerfield. 'University of Alberta, Canada

\subsection{6/OEM-2021-EPI.34}

Introduction Healthcare workers (HCW) working through the pandemic are in the front line for infection, psychological pressure and overwork.

Objectives To identify modifiable work factors associated with COVID-19 infection and mental distress, and to assess the effectiveness of provisions to mitigate their impact.

Methods A cohort study of HCWs was set up in the first weeks of the pandemic in Canada. HCWs from British Columbia, Alberta, Ontario, and Quebec completed an online questionnaire in the spring/summer of 2020, and a Phase 2 questionnaire from October 2020. They also provided a blood sample to assess SARS-CoV-2 antibodies. HCWs reporting a COVID-19 infection after the Phase 2 questionnaire were matched on job-type and province to 4 referents for a nested case-referent (C-R) study concentrating on exposures immediately prior to infection. Phase 3 is underway, with a final contact planned for March 2022.

Results 5135 HCWs completed the Phase 1 questionnaire with 93\% (4539/4857) of those eligible completing Phase 2. By March 1st 2021, 157 cases had been confirmed by PCR and a further 10 found positive only on antibody testing (an overall rate of 3.3\%). The odds of infection doubled for working one-on-one with known COVID-19 patients. Rates were lower in physicians and nurses, compared to personal support workers, health care aides, and licensed practical nurses. HCWs in a hospital setting had lower rates than those working in the community, where shortages of personal protective equipment were more widespread. High rates of anxiety (on the Hospital Anxiety and Depression Scale) were recorded in both Phase 1 and 2. Only 1 in $4 \mathrm{HCW}$ had used available mental health supports. By May 2021, 100 cases with 389 referents had been recruited to the on-going C-R study.

Conclusion Information collected prospectively has the potential to improve HCWs protection during this and future epidemics.

\section{0-294 LEVELS OF ANXIETY AND DEPRESSION AND THE PERCEIVED RISK OF COVID-19 AT WORK}

'Priscilla Ming Yi Lee, Shelly Lap-ah TSE, Dongming Wang, Yan Li, Wang Shoulin, Lau Janice Ying Chui, Shuyuan Yang, Tangchun Wu, Hongbing Shen, Xiaoming Ji, Weihong Chen. ${ }^{1}$ The Chinese University of Hong Kong, Hong Kong

\subsection{6/OEM-2021-EPI.35}

Introduction Workers who frequently contact to public or provide close-contact service may have higher anxiety and depression levels, as they may be worried about getting infected with the coronavirus at work.

Objectives To examine the association of perceived risk of COVID-19 at work (including contact with people and close contact to public) with anxiety and depression levels among non-healthcare worker, taking perceived effectiveness of company's preventive measures into account.

Methods This is a multi-city cross-sectional study in Hong Kong, Nanjing and Wuhan. We recruited 7391 non-healthcare workers who were aged $>18$ during 07/2020-04/2021. We used standardized questionnaire to collect sociodemographic, job-related information and their satisfaction of effectiveness of company's preventive measures. Participants' frequency of contact and close contact to public were collected and classified into occasionally, sometimes and often, and their anxiety and depression levels were measured using DASS-21. We performed multinomial logistic regression models to examine the association of frequency of contact and close contact to public with anxiety and depression levels. Path models were developed to analyze the potential modification of perceived effectiveness of company's preventive measures on these associations.

Results Compared with workers with occasional contact to population, workers with sometimes contact were associated with severe anxiety $(\mathrm{AOR}=1.59,95 \% \mathrm{CI}=1.27-1.99)$. The AOR for workers with often close contact to public compared with no contact were $1.53(95 \% \mathrm{CI}=1.25-1.87)$ for severe anxiety, and $1.43(95 \% \mathrm{CI}=1.14-1.79)$ for severe depression. Additionally, according to path analysis, the indirect path between contact or close contact to public and anxiety/depression were modified by perceived effectiveness of company's preventive measures.

Conclusion Workers with frequent contact with people or close contact to public was associated with worse anxiety and depressive symptoms. Companies should consider effective and sustainable measures in mitigating the risk and thereby reducing employees' anxiety and depression levels during the COVID-19 pandemic.

\section{0-369 SARS-COV-2 ANTIBODY SEROPREVALENCE AMONG FIREFIGHTERS IN ORANGE COUNTY, CALIFORNIA}

${ }^{1}$ Veronica Vieira, lan Tang, M Joseph Fedoruk, Scott Bartell, Matthew Zahn. ' University of California, Irvine, United States

\subsection{6/OEM-2021-EPI.36}

Introduction Firefighters play a vital role in Orange County (OC) California (CA) communities by assisting in emergencies, providing emergency medical treatment, and transporting ill or injured individuals, in addition to performing traditional firefighting duties. Antibody testing can be a useful tool in 
understanding seroprevalence within an occupational cohort by detecting past immune response.

Objectives We conducted serological SARS-CoV-2 antibody testing from October-November 2020 to estimate the SARS$\mathrm{CoV}-2$ seroprevalence among firefighters/paramedics in Orange County.

Methods OC firefighters employed at the time of the surveillance activity were invited to participate in a voluntary survey that collected demographic, occupational, and previous COVID-19 testing data, and a SARS-CoV-2 immunoglobulin (Ig)G antibody blood test. We collected venous blood samples using mobile phlebotomy teams that traveled to individual fire stations, in coordination with an annual tuberculosis testing campaign for firefighters employed by OC Fire Authority (OCFA), and independently for firefighters employed by cities. We estimated seroprevalence and assessed several potential predictors of seropositivity.

Results The seroprevalence was 5.3\% among 923 OCFA personnel tested, with $92.2 \%$ participating. Among firefighters self-reporting a previous positive COVID-19 test result, twenty-one $(37 \%)$ were no longer positive. There were no statistically significant differences in demographic characteristics between cases and non-cases. Work city was a significant predictor of case status $(p=0.015)$. Seroprevalence $(4.8 \%)$ was similar when aggregated across seven city fire departments (42-65\% participation). In total, 1,486 OC fire personnel were tested.

Conclusion Using a strong serosurvey design and large firefighter cohort, we observed a SARS-CoV-2 IgG seroprevalence of $5.3 \%$. The seroprevalence among OC firefighters in October 2020 was lower than the general county population estimated seroprevalence (11.5\%) in August. The difference may be due in part to safety measures taken by OC fire departments at the start of the pandemic, as well as differences in antibody test methods and/or duration of antibody response.

\section{0-481 COVID-19 INFECTION AMONG HEALTHCARE WORKERS AT MALAYSIA HOSPITALS}

'Abdul Aziz Harith, Mohd Hafiz Ab Gani, Rob Griffiths, Nor Aishah Abu Bakar, Azlihanis Abdul Hadi, Maznieda Mahjom. ${ }^{1}$ University of Otago Wellington, New Zealand

\subsection{6/OEM-2021-EPI.37}

Introduction Healthcare workers are at a substantially increased risk of being infected by COVID-19 patients. However, risk of being infected is depending on the critical phase of the pandemic, patients with COVID-19 might not be the absolute source of infection. Health workers could also be exposed to infected colleagues, infected family members, lives in communities of active transmission, or infected contacts during crowded events such as wedding reception and religious gathering.

Objectives To explore the epidemiology data of COVID-19 infection among health care workers at Malaysia Hospitals especially on patterns of transmission and characteristics.

Methods A cross-sectional surveillance study among infected COVID-19 healthcare workers working at Malaysia government hospitals.

Results 1608 healthcare staffs at hospitals have been notified with COVID-19 infection in year 2020. By proportion, nursing occupation contribute up to $40.5 \%$, followed by medical doctor (20.8\%), healthcare assistant (9.7\%), medical doctor assistant (9.1\%), medical specialist (3.2\%) and hospital administrative assistant $(2.8 \%)$. Most of cases were reported from Sabah (39.8\%), Selangor (27.5\%), Wilayah Persekutuan Kuala Lumpur \& Putrajaya (6.7\%), Sarawak (6.0\%), Perak (5.6\%) and Johor (4.7\%). By gender, seven out of ten infected healthcare staffs were female and majority of them have no comorbidity (87\%). In addition, rate of COVID-19 infection among healthcare workers was proportionately increased with rate of COVID-19 infection among community. Investigation by health authorities found 43.2\% of COVID-19 infection cause by community, $36.3 \%$ occurred between staff to staff and $17 \%$ occurred between patients to staff.

Conclusion Preponderance of infection has occurred within hospital environment. Occupational Safety and Health Unit should set up a good engagement with healthcare staff and effective strategies to protect and support the health, safety and wellbeing of staff through deep-rooted assessment of standard practice procedure especially in nursing and care activities. Digital contact tracing could improve contact tracing within hospital setting.

\section{Disease Surveillance}

\section{0-24 LUNG AND BLADDER CANCER SURVEILLANCE AMONG CONSTRUCTION WORKERS IN DIESEL ENGINE EXHAUST EXPOSED OCCUPATIONS IN ONTARIO, CANADA}

'Stephanie Ziembicki, Victoria H Arrandale, Nathan DeBono, Mamadou Dakouo, Tracy Kirkham, Paul Demers. 'Ontario Health, Canada

\subsection{6/OEM-2021-EPI.38}

Introduction Diesel engine exhaust (DEE) is a lung and bladder carcinogen and one of the most common carcinogenic exposures in Canada with over 900,000 Canadians exposed at work, according to CAREX Canada. Construction workers are an understudied group despite suspected high DEE exposure; most research on DEE has been conducted in transportation and mining industries.

Objectives This study estimates incidence rates for lung and bladder cancer in construction occupations with probable DEE exposure using the Occupational Disease Surveillance System (ODSS).

Methods The ODSS includes $\sim 2.2$ million Ontario workers identified through workers' compensation claims (1983-2014). Workers were followed for cancer diagnoses through linkage with the Ontario Cancer Registry (1964-2016). DEE-exposed construction occupations were identified using Canadian Classification Dictionary of Occupation code descriptions. Coxproportional hazards models were used to estimate hazard ratios (HR) and 95\% confidence intervals (CI), adjusted for age, birth year, and sex.

Results We identified 3980 lung and 1566 bladder cases among construction trades occupations. Compared to all other ODSS workers, construction trades occupations had small elevations in lung $(\mathrm{HR}=1.08,95 \% \mathrm{CI} 1.05-1.12)$ and bladder cancer rates $(\mathrm{HR}=1.08,1.03-1.14)$. For workers in excavating, grading, paving, and related occupations, a group expected to have high DEE exposure, positive lung cancer 